\title{
VLBI STRUCTURE OF THE QSO 3C286
}

\author{
A. J. Kus, TRAO, Torun, Poland \\ A. Marecki, TRAO, Torun, Poland \\ S. Neff, NASA/GSFC, Washington, DC, USA \\ A. van Ardenne, Radiosterrenwacht, Dwingeloo, Netherlands \\ P. N. Wilkinson, NRAL, Jodrell Bank, United Kingdom
}

\section{INTRODUCTION}

The steep, small angular size QSO 3 C286 $\left(z=0.846, m v=17^{m}\right)$ (Burbidge and Burbidge 1969) is a bright object in the radio band, which has a steep spectrum $\left(\alpha=-0.65, S \sim f^{\alpha}\right)$ from $1 \mathrm{GHz}$, peak in spectrum at about $100 \mathrm{MHz}$, and selfabsorption below $50 \mathrm{MHz}$. $3 \mathrm{C} 286$ is a member of a well established class of radio sources (the CSSQSO) and is similar to 3C147, 3C309.1, and $3 \mathrm{C} 380$ in general.

VLBI hybrid maps of 3C286 were first made by Wilkinson et al. (1979) at $609 \mathrm{GHz}$, Pearson et al. (1980) at $1671 \mathrm{MHz}$, and Simon et al. (1980) at $327 \mathrm{MHz}$. The estimate of the overall angular size and the halo component contribution were suggested to be at least 100 mas and a third of the total flux at $609 \mathrm{MHz}$, respectively. The scintillation measurements (Readhead and Hewish 1974) suggest a halo component of the order of $0.6 \times 0.3$ arcsec (Wilkinson et al. 1979).

The VLBI maps mentioned earlier made with the U.S. or global array do not contain information about a structure larger than 50 mas. The ideally suited array for this purpose is the EVN at $609 \mathrm{MHz}$, which is supplementary to the MERLIN, the U.S., and global VLBI networks.

\section{PRESENT OBSERVATIONS}

The first test observation with the EVN at $609 \mathrm{MHz}$ was conducted in August 1982 with the Mark I antenna at Jodrell Bank, one antenna at Westerbork, and, for the first time, the 15-m diameter telescope of the Torun Radio Astronomical Observatory (TRAO) at Torun, Poland. The purposes of the test were: (1) to measure the extended structure of $3 \mathrm{C} 286$, and (2) to check the performance of the network. The Mark IIc terminals were used. Jodrell Bank had a LHC polarized feed whereas Westerbork and Torun had linearly polarized feeds. The feed polarizations led to some calibration difficulties since 3C286 is highly polarized. Out of the 12 hours of observing time, we observed $3 \mathrm{C} 273 \mathrm{~B}$ as a calibrator for one hour, and four hours were lost due to TV interference at Torun. The tapes were correlated at MPIfR, and the hybrid mapping using the Jodrell Bank mapping package was done at TRAO, Torun. The $609 \mathrm{MHz}$ map is shown in Fig. 1. For comparison, see the maps made at 327,609 , and $1671 \mathrm{MHz}$ (Simon et al. 1980).

The $1420 \mathrm{MHz}, 12$-hour observing run with four EVN telescopes was performed in June 1984. In this project, the following antennas were used: the Mark I 
at Jodrell Bank, full array of the WSRT, Onsala and Torun. The data were processed at the MPIfR correlator in Bonn, and the hybrid mapping computation was initially started at Bonn but was finished in Torun. The resulting map is presented in Fig. 2. It shows core domination and a one-sided, curved jet about 150 mas long. The beam size is $23 \times 28$ mas $^{2}$. We account for all of the flux density observed at $1420 \mathrm{MHz}$.

\section{GENERAL CONCLUSIONS}

(1) The size of the source is $250 \times 300 \operatorname{mas}^{2}$ (from the $609 \mathrm{MHz}$ map), at $1420 \mathrm{MHz}$. The Gaussian component in the center dominates, the jet extends at least 150 mas $\left(750 \mathrm{~h}^{-1} \mathrm{pc}\right)$, the jet width is between 10 and 15 mas $\left(50-75 \mathrm{~h}^{-1} \mathrm{pc}\right)$. (2) Straight 80 mas $\left(400 \mathrm{~h}^{-1} \mathrm{pc}\right)$ jet at $\mathrm{PA}=222^{\circ}$ curves rapidly within $70-90$ mas $\left(50-75 \mathrm{~h}^{-1} \mathrm{pc}\right.$ ) from the core to the $\mathrm{PA}=260^{\circ}$ toward the arcsec component (van Breugel et al. 1984). (3) No counter jet is observed $\left(T_{b j e t} / T_{b c j e t} \geq 200\right)$. (4) Spectral index steepens gradually along the jet from 0.0 to -1.2 . (5) Assuming domination of the central component (deconvolved size $30 \times 12 \mathrm{mas}^{2}$ ), we find that the expected IC x-ray flux in the $0.1-3.0 \mathrm{keV}$ range is low $\left(\sim 10^{-16} \mathrm{erg} \mathrm{cm}^{-2}\right.$ $\mathrm{sec}^{-1}$ ), $B_{m e} \approx 10 \mathrm{mG}, P_{m e} \approx 10^{58}$ ergs, and the surface brightness temperature is only $2 \times 10^{10} \mathrm{~K}$. $3 \mathrm{C} 286$ therefore seems to be not as energetic as the other $3 \mathrm{C}$ sources of the class.

\section{REFERENCES}

Burbidge, G. R., and Burbidge, E. M. 1969, Nature, 222, 735.

Pearson, T. J., et al. 1980, Ap. J., 236, 714.

Readhead, A. C. S., and Hewish, A. 1974, M. N. R. A. S., 78, 1.

Simon, R. S., et al. 1980, Ap. J., 236, 707.

van Breugel, W. J. M., et al. 1984, A. J., 89, 5.

Wilkinson, P.N., et al. 1979, Ap. J., 232, 365.

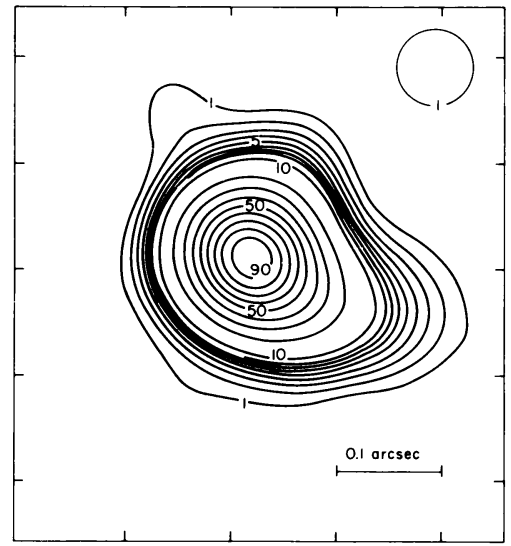

Figure 1. Hybrid map of 3C236 at 609 $\mathrm{MHz}$ (Jodrell-Westerbork-Torun). $S_{\text {max }}=18.5 \mathrm{Jy}$. $S_{\text {TOTAL }}=27 \mathrm{Jy}$. Beam $=90$ mas.

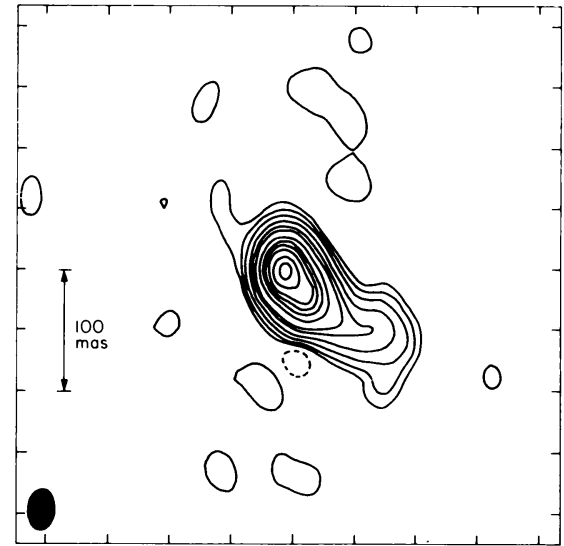

Figure 2. Map of 3C286 at $1420 \mathrm{MHz}$ (Jodrell-Westerbork-Torun-Onsala). $S_{\text {max }}=7.5 \mathrm{Jy} . S_{\text {TOTAL }}=15.7 \mathrm{Jy}$. 